\title{
Molecular cloning, sequence characterization of a novel pepper gene $N A D P-I C D H$ and its effect on cytoplasmic male sterility
}

\author{
M.H. Deng ${ }^{1,2 *}$, J.F. Wen ${ }^{3 *}$, J.L. Huo ${ }^{4 *}$, H.S. Zhu ${ }^{2}$, X.Z. Dai ${ }^{1}$, \\ Z.Q. Zhang ${ }^{1}$, H. Zhou ${ }^{2}$ and X.X. Zou ${ }^{1}$ \\ ${ }^{1}$ Institute of Vegetable Crops, Hunan Academy of Agricultural Science, \\ Changsha, China \\ ${ }^{2}$ College of Horticulture and Landscape, Yunnan Agricultural University, \\ Kunming, China \\ ${ }^{3}$ Faculty of Modern Agricultural Engineering, \\ Kunming University of Science and Technology, Kunming, China \\ ${ }^{4}$ Faculty of Animal Science and Technology, \\ Yunnan Agricultural University, Kunming, China \\ *These authors contributed equally to this study. \\ Corresponding author: X.X. Zou \\ E-mail: zou_xuexiao@163.com
}

Genet. Mol. Res. 11 (3): 3020-3031 (2012)

Received October 13, 2011

Accepted March 16, 2012

Published May 18, 2012

DOI http://dx.doi.org/10.4238/2012.May.18.15

\begin{abstract}
NADP-dependent isocitrate dehydrogenase (NADP$I C D H)$ is an important enzyme involved in energy metabolism. The complete coding sequence of the pepper (Capsicum annuum) NADP$I C D H$ gene was amplified using a reverse transcriptase PCR based on the conserved sequence information of the tomato and other Solanaceae plants and known highly homologous pepper ESTs. Nucleotide sequence analysis revealed that the pepper NADP-ICDH gene encodes a protein of 415 amino acids that has high homology with the proteins of seven species, Solanum tuberosum (100\%), Citrus limon (98\%), Daucus carota (98\%), Nicotiana tabacum (98\%), Vitis vinifera
\end{abstract}


(99\%), Arabidopsis thaliana (97\%), and Oryza sativa (98\%). Tissue expression analysis demonstrated that the pepper $N A D P-I C D H$ gene is over expressed in flower, pericarp and seed, moderately in placenta, weakly in stem and leaf, hardly expressed in root. At the abortion stages, activities and expression levels of $N A D P-I C D H$ in anthers of a sterile line were strongly reduced, while those in an $\mathrm{F}_{1}$ hybrid remained normal. Activities and expression levels of NADP-ICDH were too low to maintain balanced energy metabolism in the sterile line, which indicated that stable transcripts of $N A D P-I C D H$ are necessary to maintain energy metabolism at a normal level. When the restorer gene was transferred to the cytoplasmic male sterile line, activities and expression level of $N A D P-I C D H$ were regulated by the restorer gene and became stable. The restorer gene likely plays an important role in keeping the balance of the energy metabolism within normal levels during microspore development.

Key words: Capsicum annuum L.; NADP-isocitrate dehydrogenase; Cloning; Sequence characterization; Cytoplasmic male sterility

\section{INTRODUCTION}

Cytoplasmic male sterility (CMS) is a genetic phenomenon characterized by the absence of the production or release of viable pollen; it has been reported in more than 150 species of plants and plays an important role in the utilization of hybrid vigor (Wise and Pring, 2002). Numerous studies have shown that CMS is related to abnormal mitochondrial gene reorganization (Touzet and Budar, 2004; Linke and Börner, 2005). The reorganization of mitochondria often leads to abnormality of respiratory metabolism in CMS lines (Connettm and Hanson, 1990).

In aerobic tissues, mitochondria provide energy for cell activities (Bartoli et al., 2000; Gueguen et al., 2000; Balk and Leaver, 2001; Sweetlove et al., 2002). Numerous studies have shown that the inadequacy of energy metabolism is related to CMS (Bergman et al., 2000; Ducos et al., 2001; Sabar et al., 2003; Yui et al., 2003; Teixeira et al., 2005; Carlsson et al., 2007; Fujii et al., 2007; Sun et al., 2009).

The tricarboxylic acid (TCA) cycle is the central metabolic pathway for all aerobic processes in living organisms (Corpas et al., 1999). NADP-isocitrate dehydrogenase (NADP$I C D H$ ) catalyses the first oxidative decarboxylation reaction of the TCA cycle, yielding 2-oxoglutarate, $\mathrm{CO}_{2}$, and NADPH from isocitrate via a two-step reaction (Sienkiewicz-Porzuceka et al., 2010). NADPH can be used for reductive biosynthetic reactions, and 2-oxoglutarate can be further oxidised within the cycle or reductively aminated to glutamate. NADP-ICDH thus supplies the cell with a very important intermediate of energy metabolism, as well as precursors and reducing power for anabolic pathways (Sassi et al., 2008). The gene encoding isocitrate dehydrogenase has been cloned and sequenced from several living organisms (Fieuw et al., 1995; Gálvez et al., 1998; Camacho et al., 2002; Kirimura et al., 2002).

In the present experiment, we isolated the complete coding sequence of the pepper $N A D P-I C D H$ gene on the basis of the conserved gene sequences of tomato or other Solanaceae plant species, and subsequently performed sequence analysis. We also studied the tissue expres- 
sion of the gene $N A D P-I C D H$ and the activity and expression levels of the gene in the anthers of pepper CMS-9704A and its maintainer lines 9704B and $\mathrm{F}_{1}$, in order to establish a foundation for further understanding the action of the pepper gene $N A D P-I C D H$ and its relationship with CMS.

\section{MATERIAL AND METHODS}

\section{Sample collection, RNA extraction, and first-strand cDNA synthesis}

A typical CMS line, 9704A, its fertile maintainer line, 9704B, and a $\mathrm{F}_{1}$ hybrid line were grown in experimental fields of Yunnan Agricultural University on campus in the summer of 2011. Flowers at 4 developmental stages were selected and used for the experiments. Whole flower development was divided into 4 stages according to the progression of microsporogenesis, which was assessed by microscopic examination: stage 1, sporogenous celldivision stage; stage 2, pollen mother cell (PMC) meiosis stage; stage 3, uninucleate microspore stage; and stage 4 mature pollen stage. Fresh tissues (root, stem, leaf, flower, pericarp, placenta, and seed) were obtained for RNA isolation, frozen in liquid nitrogen, and stored at $-80^{\circ} \mathrm{C}$ until further use.

Total RNA was extracted using the RNAiso Plus (TaKaRa, Dalian, China) according to manufacturer instructions. To remove genomic DNA contamination, total RNA was digested with RNase-free DNase I (TaKaRa). Three microgram of RNA was reverse transcribed with oligo (dT) ${ }_{18}$ primer and M-MLV reverse transcriptase (Invitrogen, USA). The efficiency of reverse transcription was verified on $2 \%$ agarose gels stained with ethidium bromide.

\section{Isolation of the pepper $N A D P-I C D H$ gene}

RT-PCR was performed to isolate the pepper gene using the cDNAs of the CMS line and maintainer line from the different tissues mentioned above. The $20-\mu \mathrm{L}$ reaction system contained the following: $1.5 \mu \mathrm{L} 25 \mathrm{ng} / \mu \mathrm{L}$ cDNA, $1 \mu \mathrm{L} 2.5 \mathrm{mM}$ mixed dNTPs, $2 \mu \mathrm{L}$ 10X Taq DNA polymerase buffer ( $\mathrm{MgCl}_{2}$ plus), $0.4 \mu \mathrm{L} 10 \mu \mathrm{M}$ forward and reverse primer, $0.3 \mu \mathrm{L} 5 \mathrm{U} / \mu \mathrm{L}$ Taq DNA polymerase, and $13.4 \mu \mathrm{L}$ sterile water. The PCR program was initially started by incubation at $94^{\circ} \mathrm{C}$ for $4 \mathrm{~min}$, followed by 35 cycles of $94^{\circ} \mathrm{C}$ for $1 \mathrm{~min}, 57^{\circ} \mathrm{C}$ for $45 \mathrm{~s}, 72^{\circ} \mathrm{C}$ for $90 \mathrm{~s}$, and then $72^{\circ} \mathrm{C}$ extension for $10 \mathrm{~min}$, and finally $4^{\circ} \mathrm{C}$ to terminate the reaction. The primers for isolation of the pepper $N A D P-I C D H$ gene were designed on the basis of the conserved coding sequence data for tomato, tobacco, and potato $S C S$ genes and their highly homologous pepper ESTs. The primers for pepper $N A D P-I C D H$ were as follows: 5'-TCTTCCTCCCACTTCTCAT-3' and 5'-ACTGTCCTCTTGTCTACCG-3'. After the PCR, the gene product was cloned into a pMD18-T vector (TaKaRa) and sequenced bidirectionally with the commercial fluorometric method. At least 5 independent clones were sequenced.

\section{Bioinformatics analysis}

Sequence analysis of pepper NADP-ICDH gene was performed using the software in NCBI (http://www.ncbi.nlm.nih.gov) and ExPaSy (http://www.expasy.org). The cDNA sequences were predicted using the GenScan software (http://genes.mit.edu/GENSCAN.html). Predictions of the theoretical molecular weight $(\mathrm{Mw})$ and isoelectric point $(\mathrm{pI})$ of the putative 
protein, the signal peptide, the subcellular localization, and transmembrane topology were performed using the Compute pI/Mw Tool (http://us.expasy.org/tools/pi_tool.html), SignalP 3.0 server (http://www.cbs.dtu.dk/services/SignalP/), PSort II (http://psort.hgc.jp/), and TMHMM-2.0 server (http://www.cbs.dtu.dk/services/TMHMM-2.0/), respectively. The Blastp program and conserved domain architecture retrieval tool were used to search for similar proteins and conserved domain, respectively (http://www.ncbi.nlm.nih.gov/Blast). The alignment of the nucleotide sequences and deduced amino acid sequences were computed using ClusterX, and the phylogenetic trees were computed using the ClustalX and Mega 4.0 software with standard parameters. Secondary structures of deduced amino acid sequences were predicted with SOPMA (http://npsa-pbil.ibcp.fr/). The 3D structure was predicted on the basis of the amino acid homology modelled on a Swiss server (http://swissmodel.expasy.org/).

\section{Semi-quantitative RT-PCR}

RT-PCR was conducted to determine the expression levels of the NADP-ICDH gene in 7 pepper tissues to characterize this gene further. RT-PCR was also conducted using cDNA from the flowers in various developmental stages of CMS-9704A, maintainer lines and $\mathrm{F}_{1}$ as templates to study the relationship between the expression of the NADP-ICDH gene and CMS. To eliminate the effect of cDNA concentration, we repeated the RT-PCR 5 times using 1, 2, 3,4 , and $5 \mu \mathrm{L}$ cDNA as templates. We selected the housekeeping gene actin as the positive control. The control primers used were as follows: 5'-TGCAGGAATCCACGAGACTAC-3' and 5'-TACCACCACTGAGCACAATGTT-3'. The pepper primers used to perform the semiquantitative RT-PCR for tissue expression profile analysis were the same as those mentioned above for isolation RT-PCR. The PCRs were optimized for a number of cycles to ensure product intensity within the linear phase of amplification.

\section{Enzyme activity determinations}

NADP-ICDH activity was assayed as described in Gibon et al. (2004).

\section{Statistical analyses}

The experiments were carried out in 3 independent series with 3 replicates. Values are the mean \pm SD.

\section{RESULTS}

\section{Cloning and identification of the pepper NADP-ICDH cDNA}

Through RT-PCR of different tissue cDNAs for evaluating the expression of the pepper NADP-ICDH gene, the resulting PCR product was 1298 bp (Figure 1). cDNA nucleotide sequence analysis using the BLAST software at the NCBI server revealed that pepper NADP$I C D H$ gene was not homologous to any of the known pepper genes, and the sequences were then deposited into the GenBank database (accession No. JN885192). The sequence prediction was performed using the GenScan software and results showed that the $1248 \mathrm{bp}$ cDNA 


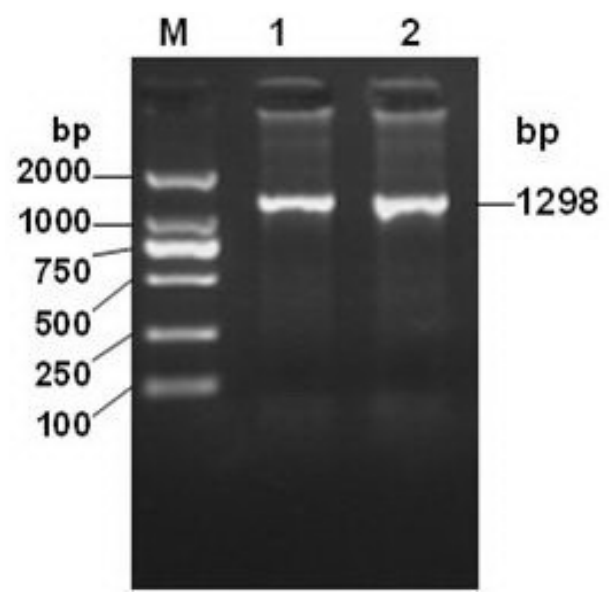

Figure 1. RT-PCR result for pepper $N A D P-I C D H$ gene. Lane $M=$ DL2000 DNA marker; lane $1=$ PCR product for pepper $9704 A$; lane $2=$ PCR product for pepper $9704 B$.

sequences represent a single gene, which encoded 415 amino acids. The cDNA sequences in the CMS line and maintainer line were exactly the same, thereby indicating that the NADP$I C D H$ gene in the CMS line was not reorganized. The complete CDS and the encoded amino acids are presented in Figure 2.

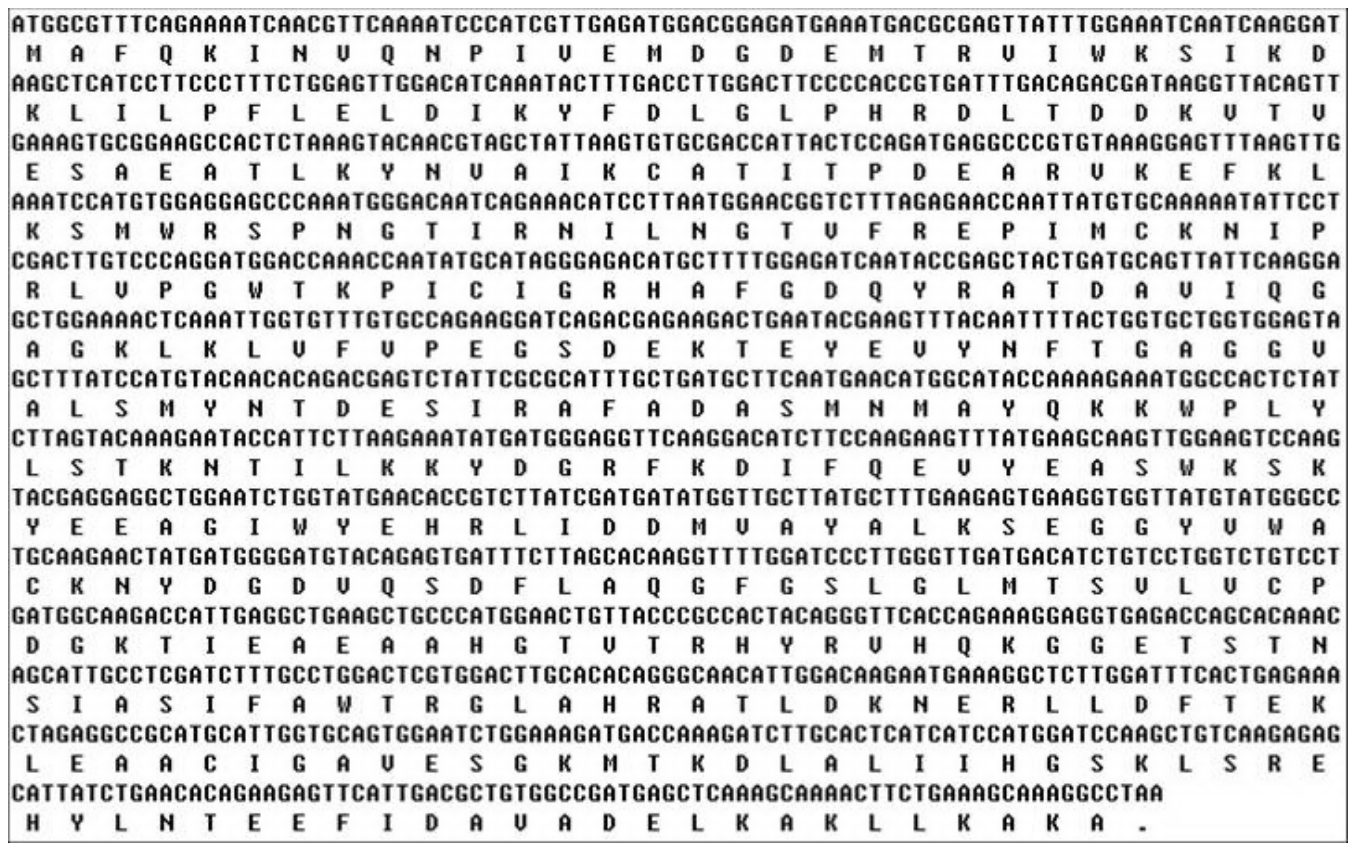

Figure 2. Complete cDNA sequence and amino acid sequence of the protein encoded by NADP-ICDH (GenBank accession No. JN885192). 


\section{Physical and chemical characteristics of pepper NADP-ICDH}

The theoretical $\mathrm{pI}$ and $\mathrm{Mw}$ were computed using the Compute $\mathrm{pI} / \mathrm{Mw}$ Tool. The $\mathrm{pI}$ and $\mathrm{Mw}$ of pepper NADP-ICDH were 6.54 and 46705, respectively. The signal peptide was predicted by SignalP 3.0 on the basis of a combination of several artificial neural networks and hidden Markov models revealed that pepper NADP-ICDH did not contain a potential signal peptide (Bendtsen et al., 2004). Using a hidden Markov model algorithm, transmembrane topology prediction made by the TMHMM program (Moller et al., 2001), showed that pepper $N A D P-I C D H$ was not a potential membrane protein. For subcellular localization analysis, the amino acid sequence was submitted to the PSORT program, and Reinhardt's method showed pepper $N A D P-I C D H$ was probably located in the peroxisome, with up to $52.4 \%$ probability (Nakai and Horton, 1999).

\section{Prediction and analysis of structures and conserved domains of pepper NADP-ICDH}

Proteins often contained several domains, each of which had their own evolutionary origins and functions. Examined using the Conserved Domain Architecture Retrieval Tool of BLAST at the NCBI server (http://www.ncbi.nlm.nih.gov/BLAST) indicated that pepper $N A D P-I C D H$ contains one separated conserved domain-Iso-dh superfamily (Figure 3).

\begin{tabular}{|c|c|c|c|c|}
\hline 75 & 225 & 375 \\
\hline & Iso_dh superfamily \\
\hline
\end{tabular}

Figure 3. Putative domains of the protein encoded by pepper NADP-ICDH.

Predictions of the secondary structure by SOPMA indicated that the deduced pepper $N A D P-I C D H$ contained 172 alpha helices, 84 extended strands, 32 beta turn, and 127 random coils (Figure 4).

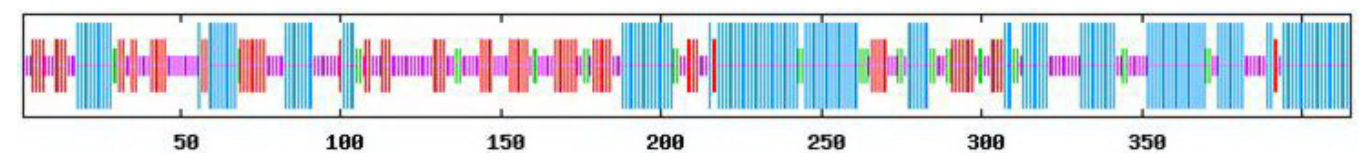

Figure 4. Secondary structure of the pepper $N A D P-I C D H$ protein predicted by S OPMA. Helices, strands and coils are indicated, respectively, with long, middle and short vertical lines.

\section{Homology modeling}

In order to better understand the detailed structure of pepper $N A D P-I C D H$, homology modeling of $N A D P-I C D H$ was performed to estimate their 3D structure (Wang et al., 2007; Benkert et al., 2011). The 3D structure of the NADP-ICDH (2-410AA) by homology modeling was similar to that of the $N A D P-I C D H$ (1 euc Chain: A). The 3D structure analysis may provide a basis for further studying the relationship between structure and function of NADP-ICDH. 


\section{Analysis of sequence identity and evolutionary relationships of pepper NADP-ICDH}

The deduced protein sequence of pepper $N A D P-I C D H$ was submitted to generate BLAST reciprocal best hits, and similarity comparison revealed that pepper $N A D P-I C D H$ protein has high homology with the NADP-ICDH proteins of 7 other species: Solanum tuberosum (100\%), Vitis vinifera (99\%), Nicotiana tabacum (98\%), Citrus limon (98\%), Daucus carota (98\%), Oryza sativa (98\%), and Arabidopsis thaliana (97\%) (Figure 5).

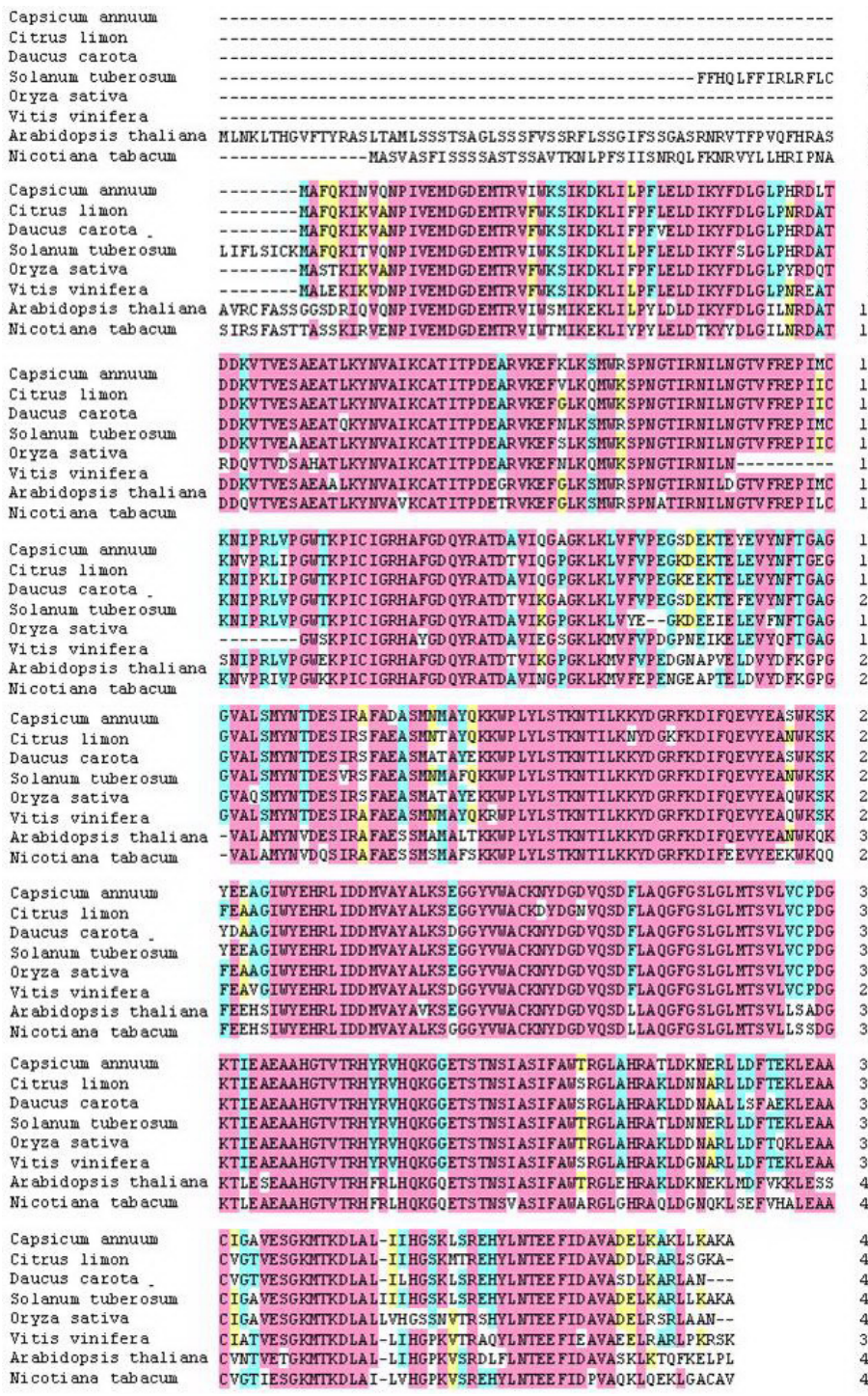

$\begin{array}{r}0 \\ 0 \\ 0 \\ 14 \\ 0 \\ 0 \\ 62 \\ 47 \\ 54 \\ 54 \\ 54 \\ 76 \\ 54 \\ 54 \\ 124 \\ 109 \\ 116 \\ 116 \\ 116 \\ 138 \\ 116 \\ 106 \\ 186 \\ 171 \\ 178 \\ 178 \\ 178 \\ 200 \\ 176 \\ 160 \\ 248 \\ 233 \\ 240 \\ 240 \\ 240 \\ 262 \\ 238 \\ 222 \\ 309 \\ 294 \\ 302 \\ 302 \\ 302 \\ 324 \\ 300 \\ 284 \\ 371 \\ 356 \\ 364 \\ 364 \\ 364 \\ 386 \\ 362 \\ 346 \\ 433 \\ 418 \\ 415 \\ 414 \\ 412 \\ 438 \\ 412 \\ 397 \\ 484 \\ 469 \\ \\ \hline\end{array}$

Figure 5. Alignment of the protein encoded by the pepper NADP-ICDH and seven other types of NADP-ICDH from Solanum tuberosum (CAA53300), Citrus limon (AF176669_1), Daucus carota (BAA34112), Nicotiana tabacum (CAA65504), Vitis vinifera (CAN83081), Arabidopsis thaliana (NP_196963), and Oryza sativa (NP_001056381). 
To evaluate the evolutionary relationships of pepper NADP-ICDH with other species, we then constructed a phylogenetic tree using DNAstar, Cluster, Mega, and the DNAman software on the basis of the NADP-ICDH amino acid sequences. The phylogenetic tree analysis revealed that the pepper $N A D P-I C D H$ gene has a closer genetic relationship with the $N A D P-I C D H$ genes of $S$. tuberosum than with those of C. limon, D. carota, N. tabacum, $V$. vinifera, A. thaliana, and $O$. sativa (Figure 6).

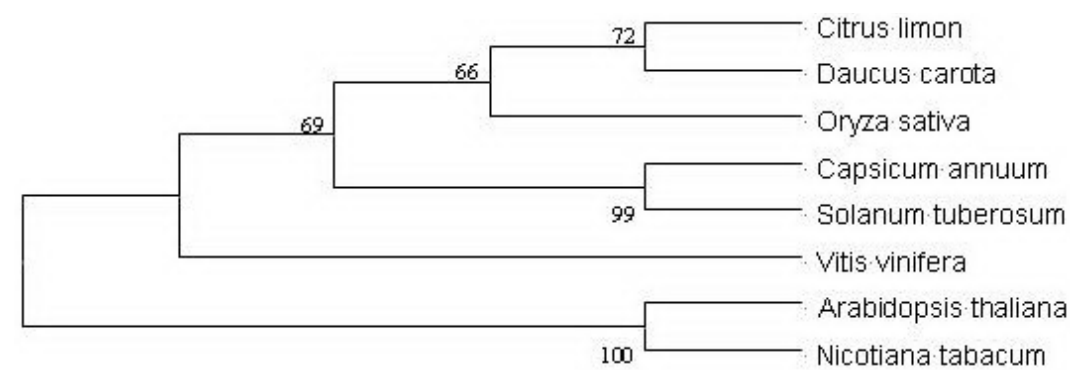

Figure 6. Phylogenetic tree for the pepper $N A D P-I C D H$ gene.

\section{mRNA tissue-specific expression profile}

To check the relative expression levels of pepper NADP-ICDH mRNA in various pepper tissues, semi-quantitative RT-PCR was performed in the 7 pepper tissues mentioned above. The continuously expressed gene, actin, was used and served as an endogenous reference for determination of targeted mRNA profiles. Results revealed that pepper NADP-ICDH gene was overexpressed in the flower, pericarp, and seed; moderately, in the placenta; weakly, in the stem and leaf; and hardly expressed, in the root (Figure 7).

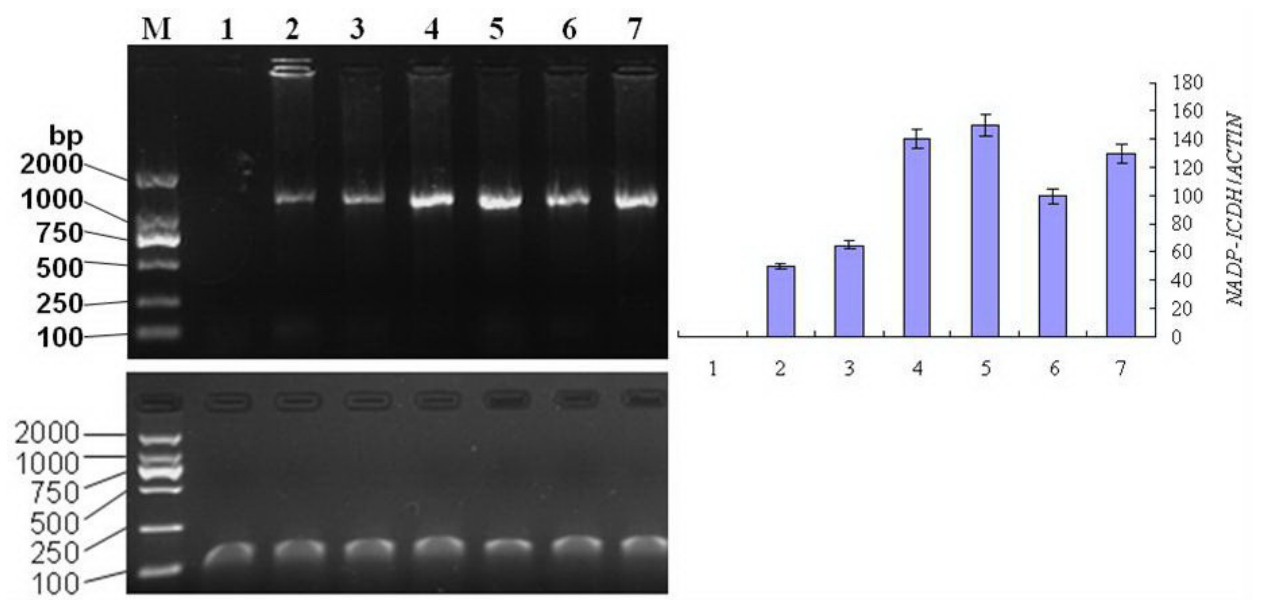

Figure 7. Tissue expression profile of the pepper NADP-ICDH gene. Lane $1=$ root; lane $2=$ stem; lane $3=$ leaf; lane 4 = flower; lane $5=$ pericarp; lane $6=$ placenta; lane $7=$ seed. The Actin expression level is used for the internal control. Lane $M=$ DL2000 DNA marker. 


\section{Activities and expression level}

The activity and expression levels of $N A D P-I C D H$ were analyzed in this study (Figure 8). Assay of $N A D P-I C D H$ activity showed that there were no more changes in the sterile line, maintainer, and hybrid $\mathrm{F}_{1}$ at stage 1 . In contrast, at stages 2,3 , and 4, the NADP-ICDH activity in the sterile line was $35.3,58.1$, and $75.0 \%$ that of the maintainer, respectively, and was $30.0,55.3$, and $70.8 \%$ that of the hybrid $\mathrm{F}_{1}$, respectively.
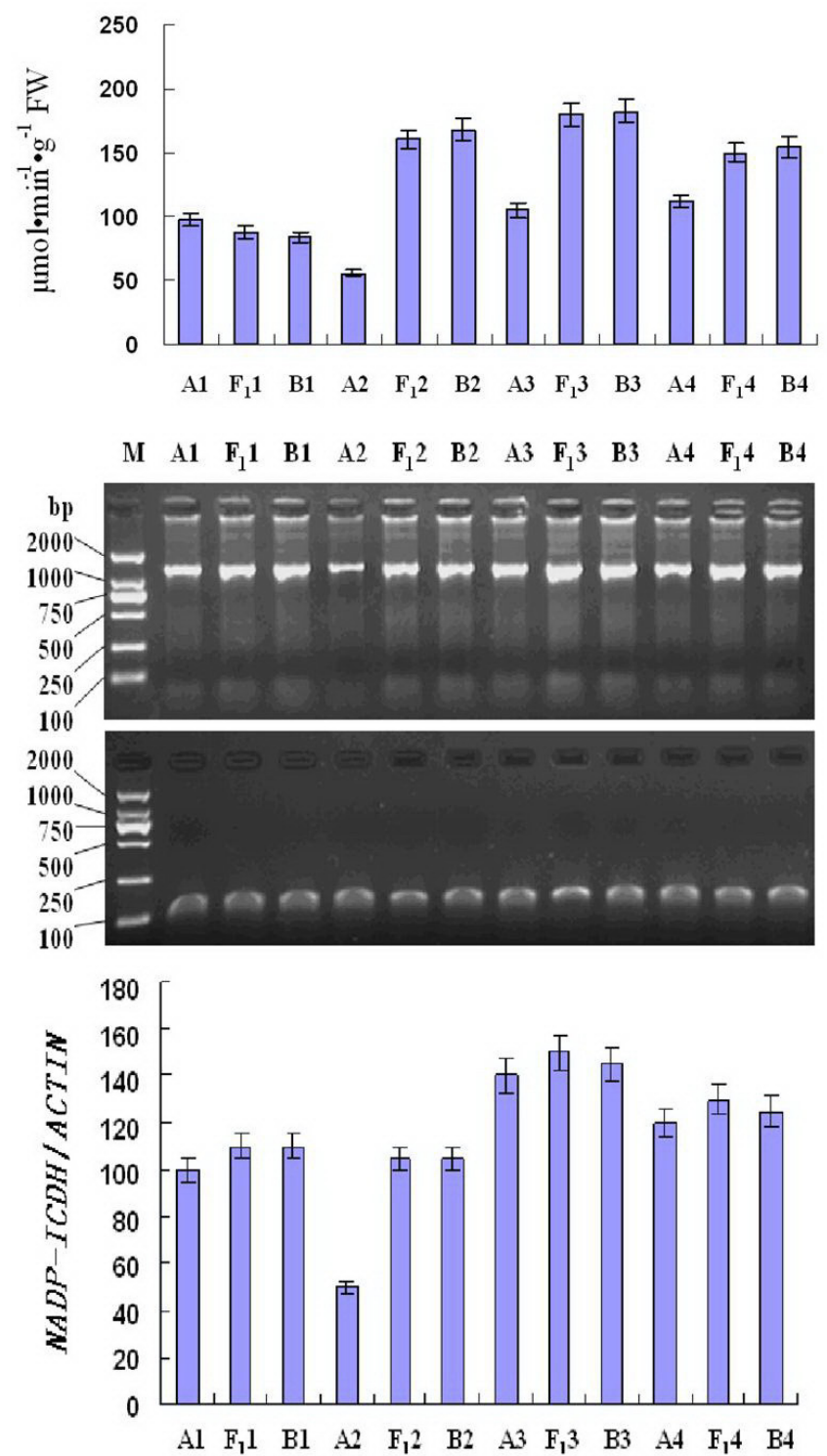

Figure 8. Activities and expression level of the pepper $N A D P-I C D H$ gene. 
Transcriptional analysis of the NADP-ICDH gene by RT-PCR was used to test the differences in the gene expression levels during the process of flower development among the sterile and fertile anthers. The results showed that there was no significant difference in the expression of $N A D P-I C D H$ in the anthers of the sterile line, maintainer, and hybrid $\mathrm{F}_{1}$ at stage 1. At stages 2, 3, and 4, expression of $N A D P-I C D H$ mRNA in the anthers of the sterile line reduced markedly, relative to the anthers of the maintainer and hybrid $\mathrm{F}_{1}$. As seen in Figure 4, the levels of actin mRNA were relatively constant in all of the RNA samples analyzed.

\section{DISCUSSION}

Reports indicate that energy demands during pollen development are very high in higher plants (Tadege and Kuhlemeier, 1997). A widely accepted hypothesis regarding the mechanism of CMS is that the increased demand for respiratory function and cellular energy in the form of ATP during anther development may be compromised by the expression of the aberrant mitochondria genes.

In aerobic tissues, mitochondria provide energy for cell activities. Numerous studies have shown that CMS is related to abnormal mitochondrial gene reorganization (Touzet and Budar, 2004; Linke and Börner, 2005).

The citric acid cycles involves a series of enzyme-catalyzed chemical reactions, which is of central importance in all living cells, especially those that use oxygen for cellular respiration. In eukaryotic cells, the citric acid cycle occurs in the matrix of the mitochondrion.

$N A D P-I C D H$ catalyzes the oxidative decarboxylation of isocitrate to 2-oxoglutarate and produces the reduced coenzyme NADPH (Gálvez and Gadal, 1995). It is the main cellular source of NADPH (Gálvez and Gadal, 1995)

Our results showed that during the abortion stage (stages 2,3, and 4), the levels of transcripts and protein activities of $N A D P-I C D H$ in the sterile line were too low to maintain a balance of energy metabolism, but they were significantly higher in the anthers of the maintainer and hybrid $\mathrm{F}_{1}$, which indicated that stable transcripts of $N A D P-I C D H$ are beneficial to maintain energy metabolism at a normal level.

Considerable evidence has revealed that nuclear gene expression can regulate mitochondrial gene expression through mitochondria-to-nucleus signaling (Karpova et al., 2002; Maxwell et al., 2002; Dutilleul et al., 2003; Rhoads et al., 2006). In our study, there was an interesting finding that during the anther abortion, unlike the sterile line, the hybrid $\mathrm{F}_{1}$ always exhibited transcripts and protein activities of $N A D P-I C D H$ that were maintained at levels consistent with those in the maintainer. This phenomenon has scarcely been reported to date. We presume that this phenomenon is probably the result of the regulation by the nuclear restorer gene during the stage of anther abortion.

\section{ACKNOWLEDGMENTS}

Research supported by the Earmarked Fund for Modern Agro-Industry Technology Research System, National Department Public Benefit Research Foundation (\#200903025), the National High Technology Research and Development Program of China (863 Program, \#2006AA100108-4-10, \#2006AA10Z1A6-3), the National Natural Science Foundation of China (Grant\#31160394), the Foundation of Natural Science of Yunnan Province, China (\#2009CD055). 


\section{REFERENCE}

Balk J and Leaver CJ (2001). The PET1-CMS mitochondrial mutation in sunflower is associated with premature programmed cell death and cytochrome c release. Plant Cell 13: 1803-1818.

Bartoli CG, Pastori GM and Foyer CH (2000). Ascorbate biosynthesis in mitochondria is linked to the electron transport chain between complexes III and IV. Plant Physiol. 123: 335-344.

Bendtsen JD, Nielsen H, von HG and Brunak S (2004). Improved prediction of signal peptides: SignalP 3.0. J. Mol. Biol. 340: 783-795.

Benkert P, Biasini M and Schwede T (2011). Toward the estimation of the absolute quality of individual protein structure models. Bioinformatics 27: 343-350.

Bergman P, Edqvist J, Farbos I and Glimelius K (2000). Male-sterile tobacco displays abnormal mitochondrial atp1 transcript accumulation and reduced floral ATP/ADP ratio. Plant Mol. Biol. 42: 531-544.

Camacho M, Rodriguez-Arnedo A and Bonete MJ (2002). NADP-dependent isocitrate dehydrogenase from the halophilic archaeon Haloferax volcanii: cloning, sequence determination and overexpression in Escherichia coli. FEMS Microbiol. Lett. 209: 155-160.

Carlsson J, Lagercrantz U, Sundstrom J, Teixeira R, et al. (2007). Microarray analysis reveals altered expression of a large number of nuclear genes in developing cytoplasmic male sterile Brassica napus flowers. Plant J. 49: 452-462.

Connett MB and Hanson MR (1990). Differential mitochondrial electron transport through the cyanide-sensitive and cyanide-insensitive pathways in isonuclear lines of cytoplasmic male sterile, male fertile, and restored petunia. Plant Physiol. 93: 1634-1640.

Corpas FJ, Barroso JB, Sandalio LM, Palma JM, et al. (1999). Peroxisomal NADP-Dependent Isocitrate Dehydrogenase. Characterization and Activity Regulation during Natural Senescence. Plant Physiol. 121: 921-928.

Ducos E, Touzet P and Boutry M (2001). The male sterile G cytoplasm of wild beet displays modified mitochondrial respiratory complexes. Plant J. 26: 171-180.

Dutilleul C, Garmier M, Noctor G, Mathieu C, et al. (2003). Leaf mitochondria modulate whole cell redox homeostasis, set antioxidant capacity, and determine stress resistance through altered signaling and diurnal regulation. Plant Cell 15: $1212-1226$

Fieuw S, Muller-Rober B, Galvez S and Willmitzer L (1995). Cloning and expression analysis of the cytosolic NADP(+)dependent isocitrate dehydrogenase from potato. Implications for nitrogen metabolism. Plant Physiol. 107: 905-913.

Fujii S, Komatsu S and Toriyama K (2007). Retrograde regulation of nuclear gene expression in CW-CMS of rice. Plant Mol. Biol. 63: 405-417.

Gálvez S and Gadal P (1995). On the function of the NADP-dependent isocitrate dehydrogenase isoenzymes in living organisms. Plant Sci. 105: 1-14.

Gálvez S, Roche O, Bismuth E, Brown S, et al. (1998). Mitochondrial localization of a NADP-dependent [corrected] isocitrate dehydrogenase isoenzyme by using the green fluorescent protein as a marker. Proc. Natl. Acad. Sci. U. S. A. 95: 7813-7818.

Gibon Y, Blaesing OE, Hannemann J, Carillo P, et al. (2004). A Robot-based platform to measure multiple enzyme activities in Arabidopsis using a set of cycling assays: comparison of changes of enzyme activities and transcript levels during diurnal cycles and in prolonged darkness. Plant Cell 16: 3304-3325.

Gueguen V, Macherel D, Jaquinod M, Douce R, et al. (2000). Fatty acid and lipoic acid biosynthesis in higher plant mitochondria. J. Biol. Chem. 275: 5016-5025.

Karpova OV, Kuzmin EV, Elthon TE and Newton KJ (2002). Differential expression of alternative oxidase genes in maize mitochondrial mutants. Plant Cell 14: 3271-3284.

Kirimura K, Yoda M, Kumatani M, Ishii Y, et al. (2002). Cloning and expression of Aspergillus niger icdA gene encoding mitochondrial NADP'-specific isocitrate dehydrogenase. J. Biosci. Bioeng. 93: 136-144.

Linke B and Börner T (2005). Mitochondrial effects on flower and pollen development. Mitochondrion 5: 389-402.

Maxwell DP, Nickels R and McIntosh L (2002). Evidence of mitochondrial involvement in the transduction of signals required for the induction of genes associated with pathogen attack and senescence. Plant J. 29: 269-279.

Moller S, Croning MD and Apweiler R (2001). Evaluation of methods for the prediction of membrane spanning regions. Bioinformatics 17: 646-653.

Nakai K and Horton P (1999). PSORT: a program for detecting sorting signals in proteins and predicting their subcellular localization. Trends Biochem. Sci. 24: 34-36.

Rhoads DM, Umbach AL, Subbaiah CC and Siedow JN (2006). Mitochondrial reactive oxygen species. Contribution to oxidative stress and interorganellar signaling. Plant Physiol. 141: 357-366.

Sabar M, Gagliardi D, Balk J and Leaver CJ (2003). ORFB is a subunit of F1F(O)-ATP synthase: insight into the basis of 
cytoplasmic male sterility in sunflower. EMBO Rep. 4: 381-386.

Sassi S, Gonzalez EM, Aydi S, Arrese-Igor C, et al. (2008). Tolerance of common bean to long-term osmotic stress is related to nodule carbon flux and antioxidant defenses: evidence from two cultivars with contrasting tolerance. Plant Soil 312: 39-48.

Sienkiewicz-Porzucek A, Sulpice R, Osorio S, Krahnert I, et al. (2010). Mild reductions in mitochondrial NAD-dependent isocitrate dehydrogenase activity result in altered nitrate assimilation and pigmentation but do not impact growth. Mol. Plant 3: 156-173.

Sun Q, Hu C, Hu J, Li S, et al. (2009). Quantitative proteomic analysis of CMS-related changes in Honglian CMS rice anther. Protein J. 28: 341-348.

Sweetlove LJ, Heazlewood JL, Herald V, Holtzapffel R, et al. (2002). The impact of oxidative stress on Arabidopsis mitochondria. Plant J. 32: 891-904.

Tadege M and Kuhlemeier C (1997). Aerobic fermentation during tobacco pollen development. Plant Mol. Biol. 35: 343-354.

Teixeira RT, Knorpp C and Glimelius K (2005). Modified sucrose, starch, and ATP levels in two alloplasmic male-sterile lines of B. napus. J. Exp. Bot. 56: 1245-1253.

Touzet P and Budar F (2004). Unveiling the molecular arms race between two conflicting genomes in cytoplasmic male sterility? Trends Plant Sci. 9: 568-570.

Wang Y, Addess KJ, Chen J, Geer LY, et al. (2007). MMDB: annotating protein sequences with Entrez's 3D-structure database. Nucleic Acids Res. 35: D298-D300.

Wise RP and Pring DR (2002). Nuclear-mediated mitochondrial gene regulation and male fertility in higher plants: Light at the end of the tunnel? Proc. Natl. Acad. Sci. U. S. A. 99: 10240-10242.

Yui R, Iketani S, Mikami T and Kubo T (2003). Antisense inhibition of mitochondrial pyruvate dehydrogenase E1 $\alpha$ subunit in anther tapetum causes male sterility. Plant J. 34: 57-66. 